ANDRÉ LUIZ da SiIVA TeIXeRA ${ }^{\dagger}$

Érica Condé Marques e OliverRA²

Marcelo Ricardo Cabral Dias ${ }^{1}$

Artigo Original

Palavras-chave

Exercício

Atividade motora

Ciclo menstrual

Distúrbios menstruais

Keywords

Exercise

Motor activity

Menstrual cycle

Menstruation disturbances

\section{Relação entre o nível de atividade física e a incidência da síndrome pré-menstrual}

\author{
Relationship between the level of physical \\ activity and premenstrual syndrome incidence
}

\section{Resumo}

OBJETIVO: Verificar a relação entre o nível de atividade física e a incidência da síndrome pré-menstrual. MÉTODOS: Trata-se de um estudo transversal realizado com 71 universitárias aparentemente saudáveis $(24,4 \pm 4,8$ anos; $61,5 \pm 8,7 \mathrm{~kg} ; 1,63 \pm 0,06 \mathrm{~m})$. O nível de atividade física foi mensurado através de questionário, e a síndrome pré-menstrual foi verificada através de um diário de sintomas respondido durante dois ciclos menstruais consecutivos. No diário são considerados 17 sintomas, os quais deveriam ser pontuados em uma escala de 5 pontos (0 a 4) de acordo com sua ocorrência, possibilitando assim o cálculo de um escore em cada ciclo. Foi considerada a ocorrência da síndrome pré-menstrual se três ou mais sintomas fossem relatados até seis dias antes da menstruação (fase pré-menstrual) e ausentes até seis dias após (fase pós-menstrual). RESULTADOS: A correlação de Spearman demonstrou uma relação significativa e negativa entre o nível de atividade física e o escore da síndrome pré-menstrual ( $r=-0,506 ;$ IC95\% -0,335 a -0,678; $p<0,001)$. Quando a amostra foi dividida em um grupo com diagnóstico positivo para síndrome pré-menstrual ( $n=31$ ) e um grupo saudável ( $n=40$ ), o teste de Mann-Whitney demonstrou que o grupo saudável possui um nível de atividade física habitual significativamente maior do que o grupo com síndrome pré-menstrual $(7,96 \pm 1,17 ; 6,63 \pm 1,20$ respectivamente) ( $p<0,001)$. CONCLUSÕES: Existe uma relação negativa entre o nível de atividade física habitual e a incidência da síndrome pré-menstrual, sendo que as mulheres com diagnóstico positivo para síndrome pré-menstrual possuem um nível de atividade física menor que as mulheres saudáveis.

\section{Abstract}

PURPOSE: To determine the relationship between the level of physical activity and the incidence of premenstrual syndrome. METHODS: A cross-sectional design was conducted on 71 apparently healthy university students $(24.4 \pm 4.8$ yrs; $61.5 \pm 8.7 \mathrm{~kg} ; 1.63 \pm 0.06 \mathrm{~m})$. The level of physical activity was determined with a questionnaire and the presence of premenstrual syndrome was verified based on daily symptoms self-reported in a diary during two consecutive menstrual cycles. 17 premenstrual symptoms are considered in the diary, which should be scored on a 5-point scale (0-4) according to their occurrence, so that a score can be calculated in each cycle. The occurrence of premenstrual syndrome was considered if three or more symptoms were reported up to six days before menstruation (premenstrual period) and were absent up to six days after menstruation (postmenstrual period). RESULTS: The Spearman correlation coefficient showed a significant and negative relationship between the level of physical activity and premenstrual syndrome score $(\mathrm{r}=-0.506$; $95 \% \mathrm{Cl}-0.335$ to $-0.678 ; \mathrm{p}<0.0011$. When the participants were divided into a group with a positive diagnosis of premenstrual syndrome $(n=31)$ and a healthy group $(n=40)$, the Mann-Whitney test showed higher habitual physical activity in the healthy group than in the premenstrual syndrome group $(7.96 \pm 1.17$ and $6.63 \pm 1.20$, respectively) $(p<0.001)$. CONCLUSIONS: There is a negative relationship between the level of physical activity and the incidence of premenstrual syndrome, with women with a positive diagnosis of premenstrual syndrome having a lower level of physical activity than healthy women.
Correspondêncic

André Luiz da Silva Teixeiro Rua Batista de Oliveira, 1.145 - Centro CEP: $36010-532$ Juiz de Fora (MG), Brasil

Recebido $17 / 01 / 2013$
Trabalho realizado no Laboratório de Fisiologia do Exercício e Avaliação Morfofuncional (LabFex) da Faculdade de Educação Física do Instituto Metodista Granbery - IMG - Juiz de Fora (MG), Brasil.

'Laboratório de Fisiologia do Exercício e Avaliação Morfofuncional - LabFex, Faculdade de Educação Física do Instituło Metodista Granbery - IMG - Juiz de Fora (MG), Brasil.

2Programa de Residência Médica em Ginecologia e Obstetrícia do Hospital Universitário, Universidade Federal de Juiz de Fora - UFJF Juiz de Fora (MG), Brasil.

Conflito de interesse: não há. 


\section{Introdução}

A síndrome pré-menstrual (SPM) é caracterizada pela ocorrência repetitiva de um conjunto de alterações físicas, do humor, cognitivas e comportamentais, que interferem negativamente nas atividades diárias, com início em torno de duas semanas antes da menstruação e alívio rápido após o início do fluxo menstrual ${ }^{1-3}$.

Em um estudo populacional, verificou-se que 96,6\% das mulheres apresentavam pelo menos 1 sintoma durante o período pré-menstrual e que $37,5 \%$ possuíam alta pontuação na escala de sintomas pré-menstruais ${ }^{4}$. No Brasil, em um estudo retrospectivo com 254 mulheres, observou-se a prevalência de $43,3 \%$ da SPM autoaferida ${ }^{5}$. Em outro estudo, a prevalência foi de 25,2\% em 1.395 mulheres entrevistadas no domicílio ${ }^{6}$. Entretanto, o instrumento mais rigorosamente elaborado para a avaliação da SPM é o Registro de Sintomas Diários (Daily Symptom Report), publicado por Freeman, DeRubeis e Rickels ${ }^{7}$, o qual leva em consideração 17 sintomas de ocorrência comum no desconforto pré-menstrual que devem ser pontuados em uma escala de 5 pontos de acordo com sua gravidade.

A SPM pode ser influenciada pela idade, pela regularidade menstrual, pela escolaridade, pelo nível socioeconômico e pelo uso de contraceptivos hormonais $s^{6,8-10}$, atingindo tanto atletas ${ }^{11,12}$, quanto não atle$\operatorname{tas}^{8,9}$. A literatura demonstra que a prática de exercícios físicos pode diminuir os sintomas pré-menstruais ${ }^{13-17}$ e, apesar de não estar bem esclarecido qual o melhor tipo (aeróbio ou resistido), a intensidade e a duração do treinamento, o American College of Obstetrician and Gynecologists ${ }^{18}$ recomenda a prática regular de exercícios físicos como um dos tratamentos não medicamentosos da SPM.

No entanto, em nossa revisão literária, não foi possível encontrar nenhum estudo associando o nível de atividade física (NAF) com a incidência da SPM. O presente estudo teve como objetivo testar as hipóteses de que: existe uma correlação negativa entre o NAF e a magnitude da ocorrência da SPM, e mulheres com diagnóstico positivo para SPM possuem um menor NAF do que as mulheres saudáveis.

\section{Métodos}

Trata-se de um estudo transversal quase experimental realizado em universitárias que foram recrutadas por conveniência. Como critério de inclusão, as participantes deveriam: ser aparentemente saudáveis; ter idade entre 18 e 35 anos e ter o ciclo menstrual regular entre 25 e 40 dias $^{19}$. Foram excluídas da amostra aquelas que: reportaram histórico de qualquer distúrbio relacionado ao sistema endócrino; haviam tido pelo menos uma gestação e as que tivessem intenção de engravidar nos três meses subsequentes ao estudo. Após os critérios de inclusão e exclusão e por desistências ao longo da coleta de dados, a amostra final foi composta por 71 mulheres.

Após concordarem em participar da pesquisa, todas assinaram um Termo de Consentimento Livre e Esclarecido que atende a resolução no 196/96 do Conselho Nacional de Saúde. A presente pesquisa foi aprovada pelo Comitê de Ética em pesquisa da Santa Casa de Misericórdia de Juiz de Fora, MG (parecer $n^{\circ}$ 011/10).

Para caracterização da amostra, foi verificado o peso e a estatura através de uma balança digital Filizolla ${ }^{\circledR} \mathrm{e}$ um estadiômetro com precisão milimétrica Sanny ${ }^{\circledR}$ respectivamente, possibilitando assim o cálculo do índice de massa corporal (IMC). As características descritivas das participantes estão apresentadas na Tabela 1 .

Para mensurar o NAF foi aplicado o questionário de Baecke, Burema e Frijters ${ }^{20}$, validado no Brasil por Florindo e Latorre ${ }^{21}$, o qual permite a obtenção de escores do NAF em três domínios: ocupacional, exercício físico/ esporte e lazer/locomoção. O domínio ocupacional leva em consideração o gasto energético durante a atividade profissional. O domínio exercício físico/esporte considera a prática sistematizada de algum esporte ou exercício físico no período de 12 meses e seu gasto energético médio. Já o domínio lazer/locomoção considera o gasto energético das atividades realizadas no tempo livre e o tempo de locomoção diária através de caminhada e/ou bicicleta. Foi considerado como valor representativo do NAF, o escore total obtido no questionário. Intencionalmente, dentro da amostra estudada havia mulheres sedentárias, recreacionalmente ativas e atletas amadoras.

Tabela 1. Características descritivas da amostra $(\mathrm{n}=71)$

\begin{tabular}{lccccc}
\hline Características & Média & Desvio-padrão & IC95\% & Mínimo & Máximo \\
\hline Idade (anns) & 24,4 & 4,8 & $23,3-25,6$ & 18 & 35 \\
Peso (kg) & 61,5 & 8,7 & $59,5-63,6$ & 42,0 & 88,0 \\
Estafura (m) & 1,6 & 0,1 & $1,6-1,7$ & 1,52 & 1,8 \\
IMC (kg/m²) & 23,0 & 2,8 & $22,3-23,7$ & 17,5 & 7 \\
Menarca (anos) & 12,2 & 1,7 & $11,8-12,6$ & 21 & 17 \\
CM (dias) & 26,8 & 2,8 & $26,1-27,5$ & 30 \\
\hline
\end{tabular}

IMC: índice de massa corporal; CM: ciclo menstrual; IC95\%: intervalo de confiança de 95\%. 


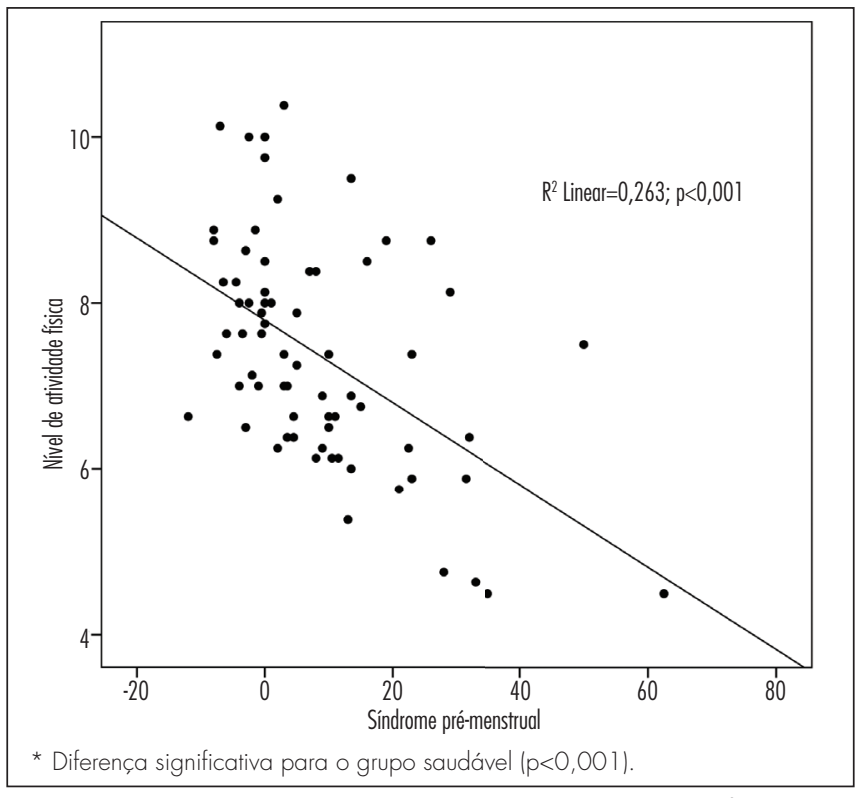

Figura 1. Correlação entre os escores do nível de atividade física e da síndrome pré-menstrual.

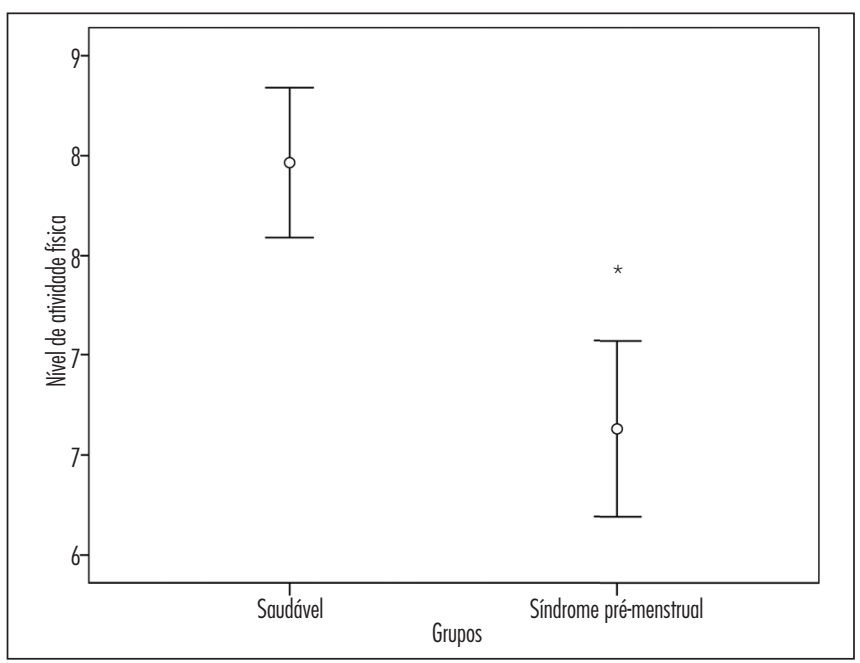

Figura 2. Nível de atividade física entre o grupo saudável e o grupo com síndrome pré-menstrual.

Tabela 2. Prevalência dos sintomas da síndrome pré-menstrual

\begin{tabular}{lc}
\hline Sintoma & Prevalência (\%) \\
\hline Ansiedade & 10,8 \\
Tensão nervosa & 10,8 \\
Irritabilidade & 9,5 \\
Choro & 7,4 \\
Insônia & 6,8 \\
Depressão & 6,1 \\
Mudanças de humor & 6,1 \\
Cefaleia & 6,1 \\
Fadiga & 6,1 \\
Dores contínuas & 5,7 \\
Cólicas & 4,6 \\
Dolorimento de seios & 4,6 \\
Inchaços & 4,6 \\
Compulsão por alimentos & 4,0 \\
Outros & 6,8 \\
\hline
\end{tabular}

Para análise da SPM, foi utilizado o critério proposto por Freeman, DeRubeis e Rickels ${ }^{7}$, o qual considera-se 17 sintomas pré-menstruais. Para tal, as voluntárias receberam uma ficha autoaplicável com o Registro de Sintomas Diários por dois ciclos menstruais consecutivos, iniciando no primeiro dia da menstruação ${ }^{12}$. Os sintomas foram pontuados em uma escala de 5 pontos ( 0 a 4 ) de acordo com sua ocorrência, sendo considerado: $0=$ ausente; $1=$ mínimo (apenas levemente aparente para você); 2 =moderado (o sintoma é perceptível, mas não altera a rotina diária); 3 =muito (continuamente incômoda pelo sintoma e/ou o sintoma interfere na atividade diária); 4 = grave (o sintoma é maior do que se pode controlar/suportar e/ou impossibilita o prosseguimento da atividade diária) ${ }^{7}$.

Foi considerada a ocorrência da SPM se três ou mais sintomas fossem relatados até seis dias antes da menstruação (fase pré-menstrual) e ausentes nos seis dias após (fase pós-menstrual). Além disso, foi calculado um escore em cada ciclo somando os valores da fase pré-menstrual e subtraindo pelos valores da fase pós-menstrual. A cada semana, foi realizada a troca de diários; e após a aplicação dos questionários, a amostra foi dividida em um grupo com diagnóstico positivo para SPM $(n=31)$ e um grupo saudável $(n=40)$.

Para a análise estatística, foi realizado o teste de normalidade de Kolmogorov-Smirnov e igualdade de variância pelo critério de Levene. Como os dados não apresentaram distribuição normal, foi utilizada uma estatística não paramétrica. Para verificar a relação entre o NAF e o escore do questionário da SPM, utilizou-se a correlação de Spearman. Para verificar a diferença no NAF entre o grupo com diagnóstico positivo para SPM e o grupo saudável, foi utilizado o teste de Mann-Whitney. Todos os dados foram analisados através do pacote estatístico SPSS 19.0 (SPSS Inc., Chicago, USA) com nível de significância de $\mathrm{p}<0,05$.

\section{Resultados}

Foi observada uma correlação fraca, porém significativa e negativa entre o NAF e o escore obtido pelo questionário de sintomas pré-menstruais $(\mathrm{r}=-0,506$; IC $95 \%-0,335$ a $-0,678 ; \mathrm{p}<0,001)$, demonstrando que quanto maior o NAF, menor é a incidência da SPM, sendo que $26,3 \%$ da variação no escore de sintomas podem ser explicados pelo NAF (Figura 1). Quando a amostra foi dividida pela ocorrência da SPM, foi verificado que as mulheres com diagnóstico de SPM $(n=31)$ possuem um NAF significativamente menor do que as mulheres saudáveis ( $\mathrm{n}=40)(\mathrm{SPM}: 6,63 \pm 1,20$; saudável: 7,96士1,17; $\mathrm{p}<0,001$ ) (Figura 2).

Adicionalmente, observou-se que os sintomas pré-menstruais com maior prevalência foram: ansiedade, tensão nervosa, irritabilidade, choro e insônia, seguidos por depressão, mudanças de humor, cefaleia e fadiga (Tabela 2). 


\section{Discussão}

O presente estudo teve como objetivo verificar a relação entre o NAF e a incidência da SPM. Os resultados confirmaram as hipóteses de que: quanto maior o NAF, menor é a magnitude dos sintomas pré-menstruais; e mulheres com diagnóstico positivo para SPM possuem um menor NAF do que as mulheres saudáveis. Esses resultados são de grande relevância clínica, pois demonstram que a prática de exercícios físicos pode ser benéfica na prevenção e/ou tratamento da SPM, corroborando assim com o posicionamento do American College of Obstetrician and Gynecologists ${ }^{18}$ que recomenda a prática regular de exercícios físicos como um dos tratamentos não medicamentosos da SPM.

Alguns estudos demonstraram que um programa de treinamento físico foi eficaz em diminuir os sintomas da $\mathrm{SPM}^{13-17}$. Prior, Vigna e Alojado ${ }^{14}$ verificaram que três meses de treinamento aeróbio reduziram os sintomas pré-menstruais em um grupo de oito mulheres previamente sedentárias. O treinamento foi composto de 2 a 4 sessões de 10 a 15 minutos de corrida por semana, sendo que foram realizados incrementos no volume e na intensidade do treinamento durante o delineamento experimental do estudo. Além disso, seis mulheres permaneceram sedentárias compondo um grupo controle, e não foram observadas diferenças significativas nos sintomas pré-menstruais no grupo que permaneceu sedentário. Outros autores demonstraram que 24 semanas de exercício aeróbio moderado (esteira, bicicleta ou escada) realizado 3 vezes na semana e intensidade entre 60 a $85 \%$ da frequência cardíaca máxima reduziram os sintomas pré-menstruais em 14 mulheres previamente sedentárias ${ }^{16}$. Nesse mesmo estudo, os autores verificaram que mulheres que são fisicamente ativas possuem menores escores de sintomas pré-menstruais do que as mulheres sedentárias ${ }^{16}$.

Uma explicação pertinente para os resultados da presente pesquisa é que a prática regular de exercícios físicos pode atenuar alguns sintomas da SPM, entre eles os sintomas de grande prevalência como ansiedade e depressão ${ }^{22-29}$. Já foi demonstrado que 12 semanas de treinamento com pesos reduziram os níveis de ansiedade em 11 sujeitos ( 6 homens e 5 mulheres) após acidente vascular isquêmico ${ }^{22}$. Curiosamente, outros autores ${ }^{23} \mathrm{di}$ vidiram 156 sujeitos em 3 grupos para o tratamento da depressão: 1) exercício; 2) medicamento; e 3) exercício e medicamento combinado. Após quatro meses de intervenção, todos os grupos apresentaram melhorias nos escores da depressão, no entanto após seis meses do término da intervenção, o grupo que fez apenas exercício apresentou menores taxas de depressão do que o grupo que recebeu o tratamento medicamentoso.

Ao contrário do que vem sendo discutido, foi encontrada uma prevalência de $71 \%$ da SPM ${ }^{11}$ em atletas de handebol. Posteriormente, também foi demonstrada uma alta prevalência da SPM (48\%) em 57 atletas de 11 modalidades esportivas, o que nos permite especular que o exercício físico quando realizado de forma exacerbada pode não ser benéfico para atenuar os sintomas da SPM. No presente estudo, como não havia atletas de alto rendimento compondo a amostra, não foi possível confirmar essa hipótese.

Como todo estudo de caráter experimental, a presente pesquisa possui algumas limitações metodológicas como a falta de controle do uso de contraceptivos hormonais. Já foi demonstrado que a SPM pode ser influenciada pelo uso de contraceptivos ${ }^{10}$. Sugere-se que pesquisas futuras sejam desenvolvidas levando em consideração o uso de agentes contraceptivos, o tipo de exercício físico e a inclusão de atletas de alto rendimento para maiores esclarecimentos sobre a relação entre o NAF e a incidência da SPM.

Em síntese, de acordo com os resultados obtidos e levando em consideração as limitações da presente pesquisa, podemos concluir que o NAF está relacionado com a incidência da SPM, sendo que quanto maior o NAF habitual, menor é a magnitude dos sintomas pré-menstruais, e ainda, mulheres com diagnóstico positivo para SPM possuem um NAF menor que as mulheres saudáveis, demonstrando assim uma preliminar evidência de que a prática regular de exercícios físicos pode auxiliar no tratamento e/ou prevenção da SPM.

\section{Referências}

1. Grady-Weliky TA. Clinical practice. Premenstrual dysphoric disorder. N Engl J Med. 2003;348(5):433-8.

2. Valadares GC, Ferreira LV, Correa Filho H, Romano-Silva MA. Transtorno disfórico pré-menstrual: revisão: conceito, história, epidemiologia e etiologia. Rev Psiquiatr Clín. 2006;33(3): 1 17-23.

3. Approbato MS, Araújo e Silva CD, Perini GF, Miranda TG, Fonseca TD, Freitas VC. [Premenstrual syndrome and school performance]. Rev Bras Ginecol Obstet. 2001;23(7):459-62. Portuguese.
4. Rasheed P, Al-Sowielem LS. Prevalence and predictors of premenstrual syndrome among college-aged women in Saudi Arabia. Ann Saudi Med. 2003;23(6):381-7.

5. Nogueira CW, Pinto e Silva JL. [Prevalence of symptoms in premenstrual syndrome]. Rev Bras Ginecol Obstet. 2000;22(6):34751. Portuguese.

6. Silva CM, Gigante DP, Carret ML, Fassa AG. Estudo populacional de síndrome pré-menstrual. Rev Saúde Pública. 2006;40(1):47-56. 
7. Freeman EW, DeRubeis R, Rickels K. Reliability and validity of a daily diary for premenstrual syndrome. Psychiatry Res. 1996;65(2):97-106.

8. Gehlert S, Song $\mathrm{IH}$, Chang $\mathrm{CH}$, Hartlage SA. The prevalence of premenstrual dysphoric disorder in a randomly selected group of urban and rural women. Psychol Med. 2009;39(1):129-36.

9. Balaha MH, Amr MA, Saleh Al Moghannum M, Saab Al Muhaidab $\mathrm{N}$. The phenomenology of premenstrual syndrome in female medical students: a cross sectional study. Pan Afr Med J. 2010;5:4.

10. Wichianpitaya J, Taneepanichskul S. A comparative efficacy of low-dose combined oral contraceptives containing desogestrel and drospirenone in premenstrual symptoms. Obstet Gynecol Int. 2013;2013:487143.

11. David AM, Di Bella ZJ, Berenstein E, Lopes AC, Vaisberg M. Incidência da síndrome pré-menstrual na prática de esportes. Rev Bras Med Esporte. 2009; 15(5):330-3.

12. Gaion PA, Vieira LF. Prevalência de síndrome pré-menstrual em atletas. Rev Bras Med Esporte. 2010;16(1):24-8.

13. Steege JF, Blumenthal JA. The effects of aerobic exercise on premenstrual symptoms in middle-aged women: a preliminary study. J Psychosom Res. 1993;37(2):127-33.

14. Prior JC, Vigna Y, Alojado N. Conditioning exercise decreases premenstrual symptoms: a prospective controlled three month trial. Eur J Appl Physiol Occup Physiol. 1986;55(4):349-55.

15. Prior JC, Vigna Y, Sciarretta D, Alojado N, Schulzer M. Conditioning exercise decreases premenstrual symptoms: a prospective, controlled 6-month trial. Fertil Steril. 1987;47(3):402-8.

16. Stoddard JL, Dent CW, Shames L, Bernstein L. Exercise training effects on premenstrual distress and ovarian steroid hormones. Eur J Appl Physiol. 2007;99(1):27-37.

17. Daley $A$. The role of exercise in the treatment of menstrual disorders: the evidence. Br J Gen Pract. 2009;59(561):241-2.

18. American College of Obstetricians and Gynecologists. Premenstrual syndrome. ACOG Pract Bull. 2000;(15).

19. Tsampoukos A, Peckham EA, James R, Nevill ME. Effect of menstrual cycle phase on sprinting performance. Eur J Appl Physiol. 2010;109(4):659-67
20. Baecke JA, Burema J, Frijters JE. A short questionnaire for the measurement of habitual physical activity in epidemiological studies. Am J Clin Nutr. 1982;36(5):936-42.

21. Florindo AA, Latorre MR. Validation and reliability of the Baecke questionnaire for the evaluation of habitual physical activity in adult men. Rev Bras Med Esporte. 2003;9(3):129-35.

22. Aidar FJ, Oliveira RJ, Silva AJ, Matos DG, Mazini Filho ML, Hickner $R C$, et al. The influence of resistance exercise training on the levels of anxiety in ischemic stroke. Stroke Res Treat. 2012;2012:298375.

23. Babyak M, Blumenthal JA, Herman S, Khatri P, Doraiswamy $M$, Moore $K$, et al. Exercise treatment for major depression: maintenance of therapeutic benefit at 10 months. Psychosom Med. $2000 ; 62(5): 633-8$

24. Herring MP, $\mathrm{O}^{\prime}$ Connor PJ, Dishman RK. The effect of exercise training on anxiety symptoms among patients: a systematic review. Arch Intern Med. 2010;170(4):321-31.

25. Ströhle A. Physical activity, exercise, depression and anxiety disorders. J Neural Transm. 2009; 1 16(6):777-84.

26. Pereira DS, Queiroz BZ, Miranda AS, Rocha NP, Felício DC, Mateo EC, et al. Effects of physical exercise on plasma levels of brain-derived neurotrophic factor and depressive symptoms in elderly women - a randomized clinical trial. Arch Phys Med Rehabil. 2013. pii: S0003-9993(13)0030 doi:10.1016/i. apmr.2013.03.029. [Epub ahead of print].

27. Smits JA, Zvolensky M, Rosenfield D, Marcus BH, Church TS, Frierson GM, et al. The efficacy of vigorous-intensity exercise as an aid to smoking cessation in adults with elevated anxiety sensitivity: study protocol for a randomized controlled trial. Trials. 2012;13:207.

28. Stanton R, Reaburn P. Exercise and the treatment of depression: a review of the exercise program variables. J Sci Med Sport. 2013. pii: S1440-2440(13)00058-3. doi: 10.1016/i.jsams.2013.03.010. [Epub ahead of print]

29. Greenwood BN, Strong PV, Loughridge AB, Day HE, Clark PJ, Mika $A$, et al. $5-\mathrm{HT}_{2 C}$ receptors in the basolateral amygdala and dorsal striatum are a novel target for the anxiolytic and antidepressant effects of exercise. Plos One. 2012;7(9):e46118. 